\title{
Secondary Malignant Peritoneal Mesothelioma of the Greater Omentum after Therapy for Primary Pleural Mesothelioma
}

\author{
Andreas Gutzeit ${ }^{a-c} \quad$ Carolin Reischauer $^{a} \quad$ Klaus Hergan $^{c} \quad$ Sebastian Kos ${ }^{b}$ \\ Justus E. Roos ${ }^{\mathrm{a}}$ \\ ${ }^{a}$ Department of Radiology, Cantonal Hospital Winterthur, Winterthur, and \\ ${ }^{b}$ Department of Radiology, Research Group, Klinik St. Anna, Lucerne, Switzerland; \\ ${ }^{\mathrm{c}}$ Department of Radiology, Paracelsus Medical University Salzburg, Salzburg, Austria
}

\section{Key Words}

Secondary malignant mesothelioma $\cdot$ Pleural mesothelioma $\cdot$ Greater omentum

\begin{abstract}
Mesothelioma is the most common malignant primary tumor of the pleura and usually associated with inhalation of asbestos fibers. In contrast, peritoneal mesothelioma is a rare entity whose pathomechanism is not yet fully understood. The coexistence of pleural mesothelioma with secondary involvement of the abdominal cavity has not been addressed in the literature. In this case report, we describe secondary malignant mesothelioma of the greater omentum. A 69-year-old man with histologically proven pleural mesothelioma on the right side and no past medical history of asbestos exposure received palliative treatment consisting of a talc pleurodesis. After a 6-month interval of stable disease, a local progressive tumor of the right pleura was seen on a CT scan. Eleven months later, during follow-up, the patient presented at our emergency department with a sudden onset of diffuse abdominal pain. Abdominal ultrasound revealed a mass within the greater omentum and the coexistence of free fluid. Subsequent abdominal CT scans demonstrated tumor infiltration from the right pleura by a transdiaphragmatic route into the abdomen, where diffuse infiltration of the greater omentum was observed. Aspiration of the ascites and the biopsy of the greater omentum confirmed the diagnosis of secondary malignant mesothelioma of the peritoneum. In conclusion, we present the extremely rare diagnosis of secondary malignant mesothelioma of the abdomen, which arose as a result of local progression from the right pleura into the abdomen.
\end{abstract}


Gutzeit et al.: Secondary Malignant Peritoneal Mesothelioma of the Greater Omentum after Therapy for Primary Pleural Mesothelioma

\section{Introduction}

The most common malignant mesothelioma arises from mesothelial cells of the pleura. A rare manifestation of mesothelioma is a primary tumor originating from the peritoneum [1]. Only a few cases of peritoneal mesothelioma have been reported in the literature [2-4], and all publications described primary peritoneal mesothelioma without the coexistence of other tumors, especially pleural mesothelioma [4].

We report an unusual case of secondary malignant mesothelioma, which originated from locally progressive pleural mesothelioma and extended into the abdomen by infiltration of the diaphragm and, ultimately, of the greater omentum.

\section{Case Report}

A 69-year-old man with histologically proven epithelioid-type primary malignant pleural mesothelioma of the right thorax was initially diagnosed by CT imaging. A localized tumor of the right pleura, without any mediastinal lymphadenopathy, pulmonary metastasis or abdominal involvement, was depicted (fig. 1). Due to progression of the disease and the patient's request, palliative treatment of the coexisting large effusion included right-sided talc pleurodesis. Follow-up CT scans showed a slowly enlarging pleural tumor which was initially limited to the thoracic space. Eleven months after the diagnosis, the patient presented at our emergency department with a sudden onset of diffuse abdominal pain. An initial ultrasound examination revealed infiltration of the greater omentum, which was interpreted as omental infiltration or 'omental cake' (fig. 2). CT scans of the thorax and abdomen confirmed the diffuse tumor infiltration of the greater omentum as well as the free fluid in all 4 abdominal quadrants (fig. 3). Interestingly, the origin of the abdominal tumor was progressive infiltration of the right pleural mesothelioma through the right diaphragm into the abdomen and the greater omentum (fig. 4). One day after confirming the CT results, an ultrasound-guided biopsy of the tumorous infiltration of the greater omentum was performed. The fine needle aspiration revealed malignant mesothelial cells, which were similar to the aspirated cells in the right pleura (fig. 5). Biopsy confirmed the malignant mesothelioma had the same histological architecture as seen in the patient's pleural mesothelioma (fig. 6).

\section{Discussion}

Malignant mesothelioma arises from the mesothelial cells of any serosal membranes and is most commonly found in the pleura [5]. In rare circumstances, it can also originate in other anatomical regions, including the serosal of the pericardium, tunica vaginalis and, exceedingly rarely, the peritoneum. In the USA, only 250 new cases of malignant primary peritoneal mesothelioma are diagnosed per year, which indicates that this is a very rare diagnosis [6].

All data in the literature describe peritoneal mesothelioma as being primary peritoneal mesothelioma, and apparently all cases are associated with asbestos exposure [7-9]. The pathomechanism is not fully understood. One theory is that asbestos fibers protrude from the lung surface after inhalation and cause repeated cycles of irritation in the parietal mesothelial cells, which leads to primary peritoneal mesothelioma [5]. Another explanation concerns the expectoration of inhaled asbestos fibers swallowed beforehand. After being 
expectorated, the fibers penetrate the bowel wall and end up in the lymphatic and splanchnic circulation [10]. Imaging features associated with primary malignant mesothelioma include nonspecific findings of ascites, omental thickening and smooth contrast enhancement of the peritoneum [8], which lead to a wide range of differential diagnoses such as metastatic peritoneal carcinomatosis, peritonitis or omental infarction $[3,8]$.

This case report describes the unique case of histologically proven pleural mesothelioma with local progression and secondary infiltration of the abdominal cavity, especially of the greater omentum. Interestingly, this patient had no known previous asbestos exposure, and no abdominal tumor manifestation was present at the time of pleural mesothelioma diagnosis. Based on our data, we draw the conclusion that a secondary, transdiaphragmatic tumorous infiltration of pleural mesothelioma into the abdominal cavity caused the presence of secondary malignant peritoneal mesothelioma. To our knowledge, there are no other descriptions of secondary peritoneal mesothelioma in the literature. In particular, we did not find any cases with invasion of the greater omentum as a secondary infiltration of pleural mesothelioma. We assume that infiltration by pleural mesothelioma into the abdomen is very rare, because in most circumstances the disease's aggressiveness leads to the premature death of the patient before such a local progression could occur.

The ultrasound and CT imaging features included a diffuse infiltration of the greater omentum with associated ascites in all 4 abdominal quadrants. From an abdominal point of view, the changes are similar to those in metastatic peritoneal carcinomatosis, tuberculous peritonitis, omental infarction or even so-called primary serous carcinoma of the peritoneum. However, in combination with the local progression of pleural mesothelioma, our first differential diagnosis was locally progressive pleural mesothelioma, which infiltrated the abdominal space as histologically and cytologically documented.

In conclusion, pleural mesothelioma could end in local progression into the abdomen. Although tumor infiltration above and beyond the diaphragm is extremely rare, it is important to be aware of such tumor progression, and the presence of secondary peritoneal mesothelioma should be considered.

\section{Acknowledgement}

We want to thank the Department of Pathology, Cantonal Hospital Winterthur, for helping with the preparation of the histological image.

\section{References}

1 Asensio JA, Goldblatt P, Thomford NR: Primary malignant peritoneal mesothelioma. A report of seven cases and a review of the literature. Arch Surg 1990;125:1477-1481.

-2 Liu YC, Kuo YL, Yu CP, Wu HS, Yu JC, Chen CJ, Chan DC, Yu CY, Hsieh CB, Chen TW: Primary malignant mesothelioma of the greater omentum: report of a case. Surg Today 2004;34:780-783.

-3 Shin MK, Lee OJ, Ha CY, Min HJ, Kim TH: Malignant mesothelioma of the greater omentum mimicking omental infarction: a case report. World J Gastroenterol 2009;15:4856-4859.

4 Robinson BW, Lake RA: Advances in malignant mesothelioma. N Engl J Med 2005;353:1591-1603.

5 Jeong YJ, Kim S, Kwak SW, Lee NK, Lee JW, Kim KI, Choi KU, Jeon TY: Radiograhics 2008;28:801-817.

-6 Price B, Ware A: Mesothelioma trends in the United States: an update based on Surveillance, Epidemiology, and End Results Program data for 1973 through 2003. Am J Epidemiol 2004;159:107-112.

-7 Manzini Vde P, Recchia L, Cafferata M, Porta C, Siena S, Giannetta L, Morelli F, Oniga F, Bearz A, Torri V, Cinquini M: Malignant peritoneal mesothelioma: a multicenter study on 81 cases. Ann Oncol 2010;21:348353.

8 Robinson BW, Lake RA: Advances in malignant mesothelioma. N Engl J Med 2005;353:1591-1603. 


\section{Case Reports in Oncology}

\begin{tabular}{l|l}
\hline Case Rep Oncol 2013;6:236-241 \\
\hline DOI: 10.1159/000351124 & $\begin{array}{l}\text { ○ 2013 S. Karger AG, Basel } \\
\text { www.karger.com/cro }\end{array}$ \\
\hline
\end{tabular}

Gutzeit et al.: Secondary Malignant Peritoneal Mesothelioma of the Greater Omentum after Therapy for Primary Pleural Mesothelioma

9 Kebapci M, Vardareli E, Adapinar B, Acikalin M: CT findings and serum ca 125 levels in malignant peritoneal mesothelioma: report of 11 new cases and review of the literature. Eur Radiol 2003;13:2620-2626.

10 Skucas J: Peritoneum, mesentery, and extraperitoneal soft tissue; in Skucas J (ed): Advanced Imaging of the Abdomen. London, Springer-Verlag, 2005, pp 865-932.

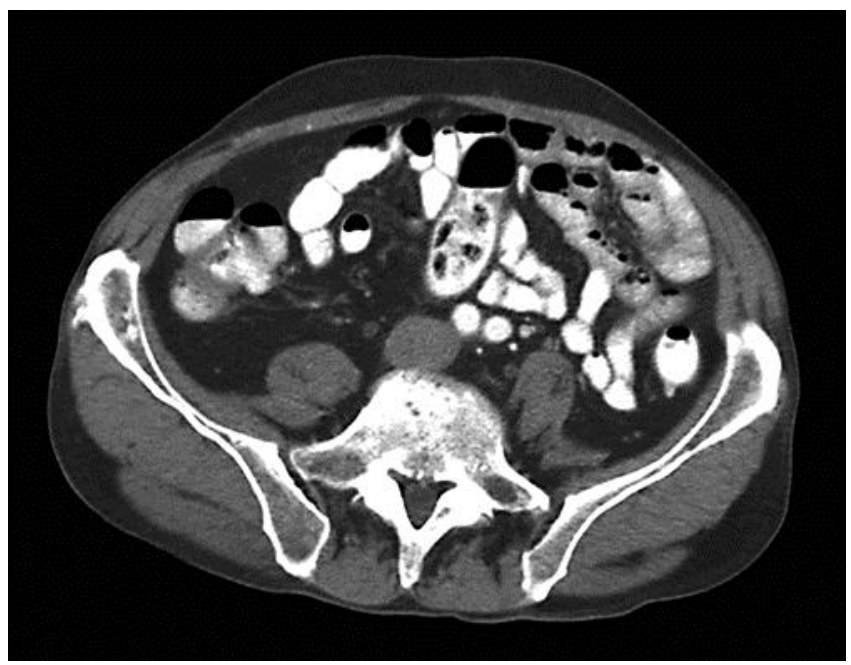

Fig. 1. Initial axial CT at the mid-abdominal level reveals no infiltration of the greater omentum and absence of ascites.

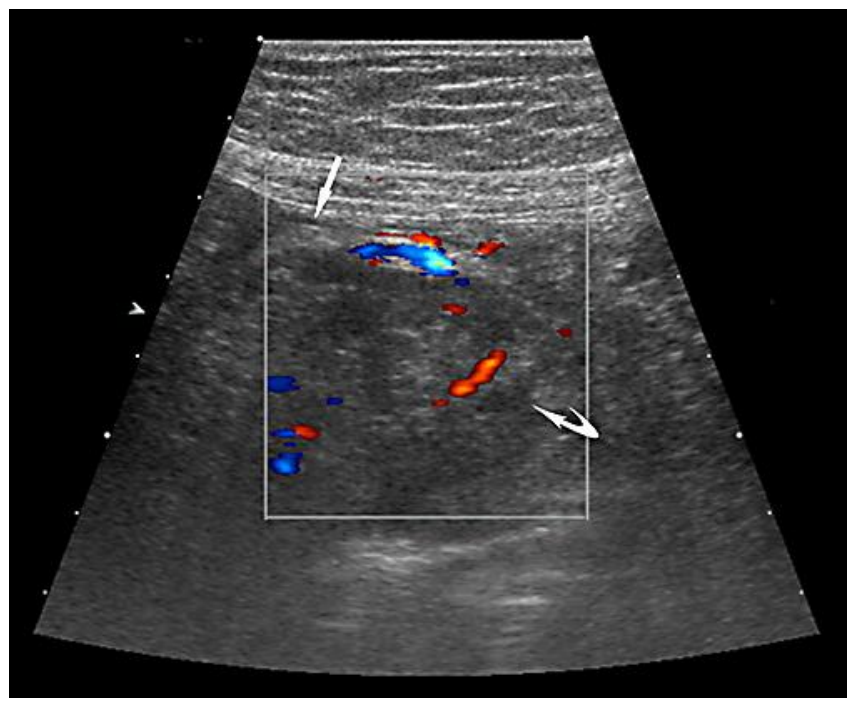

Fig. 2. Abdominal ultrasound, performed 11 months after the diagnosis of pleural mesothelioma, shows a diffuse and large infiltration of the greater omentum (curved arrow) with close proximity to the peritoneum (straight arrow). Color Doppler ultrasound depicts a moderate vascularity of the tumor mass. 


\section{Case Reports in Oncology}

\begin{tabular}{l|l}
\hline Case Rep Oncol 2013;6:236-241 & \\
\hline DOI: $10.1159 / 000351124$ & $\begin{array}{l}\text { C 2013 S. Karger AG, Basel } \\
\text { www.karger.com/cro }\end{array}$ \\
\hline
\end{tabular}

Gutzeit et al.: Secondary Malignant Peritoneal Mesothelioma of the Greater Omentum after Therapy for Primary Pleural Mesothelioma

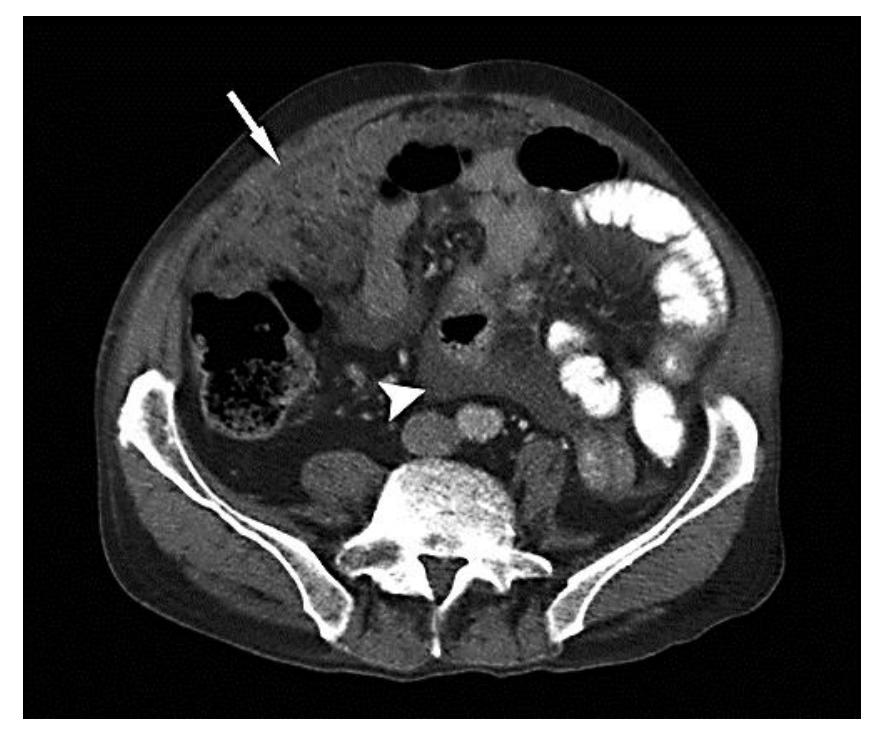

Fig. 3. Follow-up axial CT of the abdomen confirms the tumorous infiltration of the greater omentum (known as omental cake, arrow) and ascites (arrowhead), as could be seen on the preceding ultrasound.

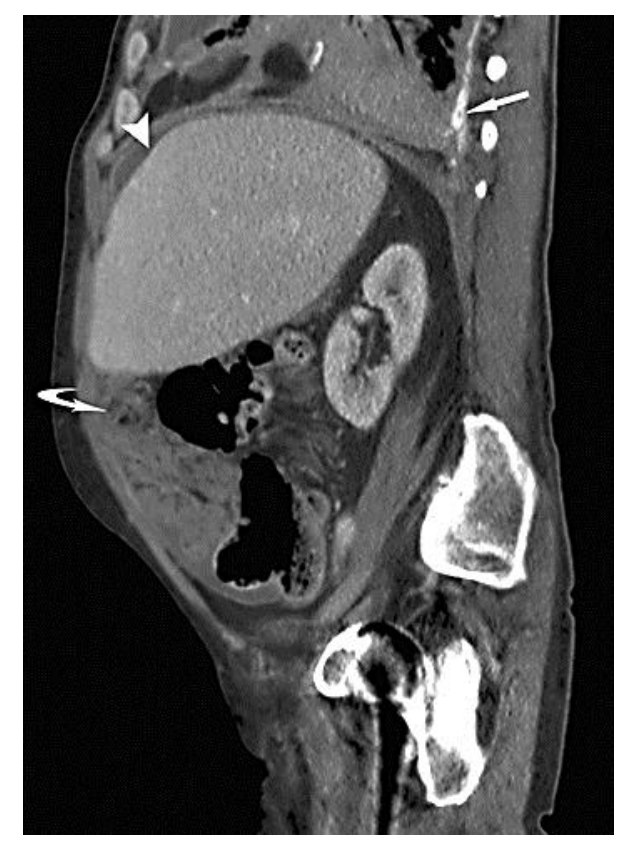

Fig. 4. Sagittal reformation of the middle abdomen demonstrates the diffuse infiltration extending from the right pleural space (arrow) and abdominal cavity. Infiltration of the greater omentum can be seen with typical confirmation of the omental cake (curved arrow). Additionally, free infradiaphragmatic fluid is depicted (arrowhead). 
Gutzeit et al.: Secondary Malignant Peritoneal Mesothelioma of the Greater Omentum after Therapy for Primary Pleural Mesothelioma

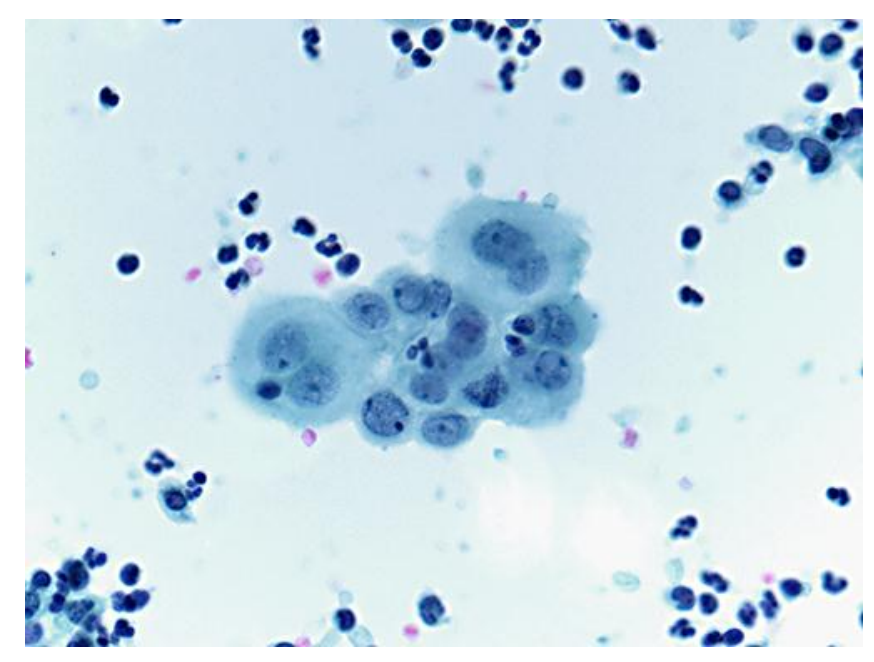

Fig. 5. Aspiration of ascites shows atypical mesothelial cells, originating from the primary tumor in the right pleura, consistent with malignant secondary peritoneal mesothelioma.

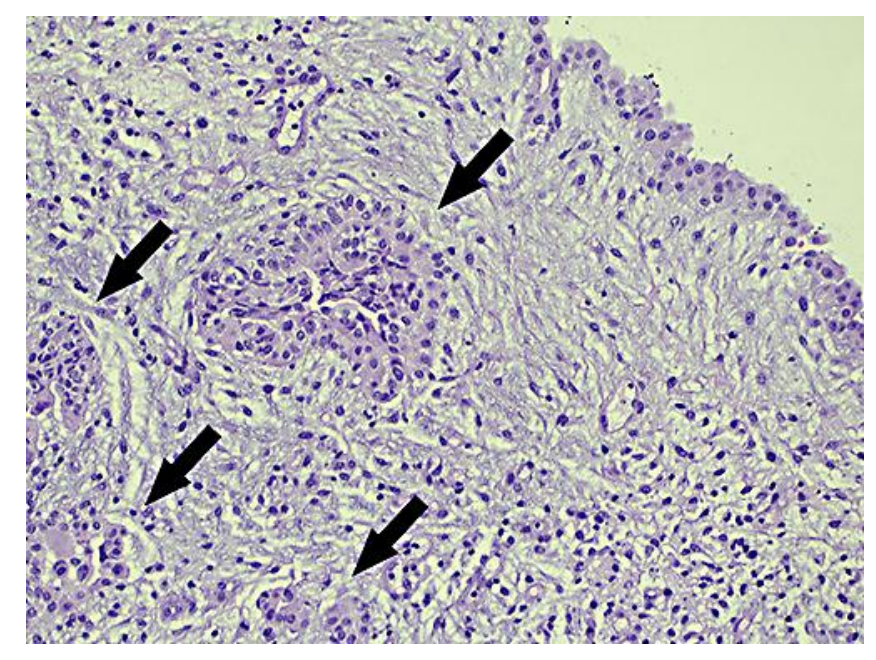

Fig. 6. Histological analysis (HE staining) shows infiltrating malignant mesothelioma (arrows) within the greater omentum. In combination with the cytological results, secondary peritoneal mesothelioma, originating from the previously diagnosed pleural mesothelioma with infiltration of the abdominal space, was confirmed. 